\title{
Phân tích hiệu quả kinh tế trong sản xuất táo của nông hộ theo mô hình nhà lưới tại huyện Ninh Phước, tỉnh Ninh Thuận
}

\section{Analyzing economic efficiency of apple farmers in the envelop house model in Ninh Phuoc district, Ninh Thuan province}

\author{
Đặng Tường Anh Thư ${ }^{1 *}$, Nguyễn Thị Trà ${ }^{1}$, Nguyễn Hữu Lộc ${ }^{1}$, Phạm Trung Hậu ${ }^{1}$, Trần Hoài Nam ${ }^{1}$ \\ ${ }^{1}$ Trường Đại học Nông Lâm Thành phố Hồ Chí Minh, Việt Nam \\ *Tác giả liên hệ, Email: dangtuonganhthu@gmail.com
}

\section{THÔNG TIN}

DOI:10.46223/HCMCOUJS.

Ngày nhận: 25/03/2021

Ngày nhận lại: 14/05/2021

Duyệt đăng: 03/06/2021

Tù khóa:

canh tác táo; hiệu quả kinh tế; huyện Ninh Phước; mô hình nhà lưới

Keywords:

apple production; econimic efficiency; Ninh Phuoc district; envelop house model

\section{TÓM TẮT}

Mô hình nhà lưới trong canh tác táo là phương pháp bảo vệ hiệu quả trước sự tấn công của sâu bệnh và đang được áp dụng rộng rãi trong những năm gần đây. Trong nghiên cứu này, phương pháp màng bao dữ liệu (Data Envelopment Analysis - DEA) được sử dụng nhằm phân tích hiệu quả kĩ thuật, hiệu quả phân phối nguồn lực, hiệu quả chi phí và hiệu quả quy mô của nông hộ sản xuất táo tại huyện Ninh Phước, tỉnh Ninh Thuận. Số liệu được thu thập từ 240 hộ trồng táo trên địa bàn huyện Ninh Phước. Kết quả nghiên cứu cho thấy nông hộ sản xuất táo theo mô hình nhà lưới có hiệu quả kỹ thuật (0.962) rất cao nhưng hiệu quả phân phối nguồn lực (0.741) và hiệu quả sử dụng chi phí (0.713) ở mức trung bình. Mặt khác, khi áp dụng mô hình nhà lưới thì nông hộ trồng táo tăng hiệu quả tài chính gấp 1.87 lần so với hộ không áp dụng và giảm chi phí sản xuất cũng như giảm lượng lãng phí các yếu tố đầu vào trong quá trình sản xuất.

ABSTRACT
In recent years, the envelope house model is a development
and the protection method is of the attacking insect. The research
used Data Envelopment Analysis (DEA) method to evaluate the
technical efficiency, resource allocative efficiency, cost efficiency,
and scale efficiency of apple production in Ninh Phuoc district,
Ninh Thuan province. Data were collected by interviewing 240
farmers apple in the Ninh Phuoc district. The results of the research
showed that the farmer's apple had a relatively high level of
technical efficiency ( 0.962$)$, an average level of resource allocative
efficiency (0.741), and cost efficiency (0.713). The results also
showed that the applied farmers model has increased the
productive efficiency 1.87 times of the not applied farmers model
and decreased input factors in production.

\section{1. Đặt vấn đề}

Ninh Thuận là tỉnh thuộc vùng duyên hải Nam Trung Bộ có lượng mưa bình quân nhiều năm khoảng 1,100mm. Trong giai đoạn từ năm 2008 - 2018, những thay đổi về nhiệt độ, lượng 
mưa, mực nước ngầm đã gây ra tình trạng khô hạn ở khu vực với mức độ rất nghiêm trọng (71.1\%) gây thiệt hại đến hoạt động sản xuất nông nghiệp của nông hộ (N. H. Tran \& Le, 2019). Tuy nhiên, đây cũng là một lợi thế để tỉnh Ninh Thuận phát triển một số cây trồng đặc thù như nho, táo, măng tây, hành và tỏi. Từ lâu cây táo đã gắn bó với người nông dân nơi đây do đặc tính dễ trồng, dễ chăm sóc nên loại cây này phát triển thuận lợi với diện tích khoảng 1,100 ha và được trồng chủ yếu tại huyện Ninh Phước, Ninh Hải, Ninh Sơn và Thành phố Phan Rang - Tháp Chàm với nhiều giống táo mới có năng suất và chất lượng cao (Sở NN \& PTNT tỉnh Ninh Thuận, 2020). Trong sản xuất nông nghiệp, hiệu quả kinh tế là nhân tố quan trọng trong phát triển sản xuất, đặc biệt là ở các nước đang phát triển nơi mà nguồn lực của người dân còn nhiều hạn chế. Tuy nhiên, những nước này có thể hưởng lợi từ việc nghiên cứu hiệu quả, từ đó có thể nâng cao năng suất bằng việc nâng cao hiệu quả mà không cần tăng thêm nguồn lực hay phát triển công nghệ mới (Ali \& Byerlee, 1991).

Mặt khác, khó khăn lớn nhất hiện nay trong canh tác táo là việc kiểm soát dịch bệnh vì táo rất dễ bị sâu bệnh, chim, côn trùng tấn công nhất là ruồi vàng, điều này đã ảnh hưởng rất lớn đến hiệu quả trong canh tác táo của nông hộ. Những năm gần đây, nông dân trong tỉnh đã sử dụng nhiều biện pháp kỹ thuật nhằm phòng trừ sự tấn công của ruồi vàng, trong đó phương pháp bao lưới cho vườn táo là phương pháp hiệu quả mà nhiều nông hộ đang áp dụng. Vì vậy, mục tiêu của nghiên cứu này là phân tích hiệu quả kinh tế trong sản xuất táo của nông hộ theo mô hình nhà lưới tại huyện Ninh Phước, tỉnh Ninh Thuận, từ đó gợi ý một số khuyến nghị nhằm nâng cao hiệu quả kinh tế trong sản xuất táo.

\section{Co' sở lý thuyết}

Theo Farrell (1957), hiệu quả kinh tế (Economic Efficiency - EE) là thước đo kết quả tổng hợp của nhà sản xuất và bằng với tích số của hiệu quả kỹ thuật (Technical Efficiency - TE) và hiệu quả phân phối (Alocative Efficiency - AE) hay EE = TE x AE. Trong đó, hiệu quả kỹ thuật (TE) là khả năng sản xuất ra một mức đầu ra cho trước từ một tập hợp đầu vào nhỏ nhất, hay khả năng tạo ra một lượng đầu ra tối đa từ một lượng đầu vào cho trước, ứng với một trình độ công nghệ nhất định. Hiệu quả phân phối (AE) là khả năng lựa chọn được một lượng đầu vào tối ưu mà ở đó giá trị sản phẩm biên của đơn vị đầu vào cuối cùng bằng với giá của đầu vào đó.

Hình 1 minh họa phương pháp để đo lường TE, $\mathrm{AE}$ và EE. Cụ thể, khi một đơn vị sản xuất tại điểm $\mathrm{P}$, giá trị ước lượng của $\mathrm{TE}, \mathrm{AE}$ và $\mathrm{EE}$ tương ứng tại điểm này được tính toán như công thức sau: $\mathrm{TE}=0 \mathrm{Q} / 0 \mathrm{P} ; \mathrm{AE}=0 \mathrm{R} / 0 \mathrm{Q} ; \mathrm{EE}=\mathrm{TE} * \mathrm{AE}=0 \mathrm{R} / 0 \mathrm{P}$.

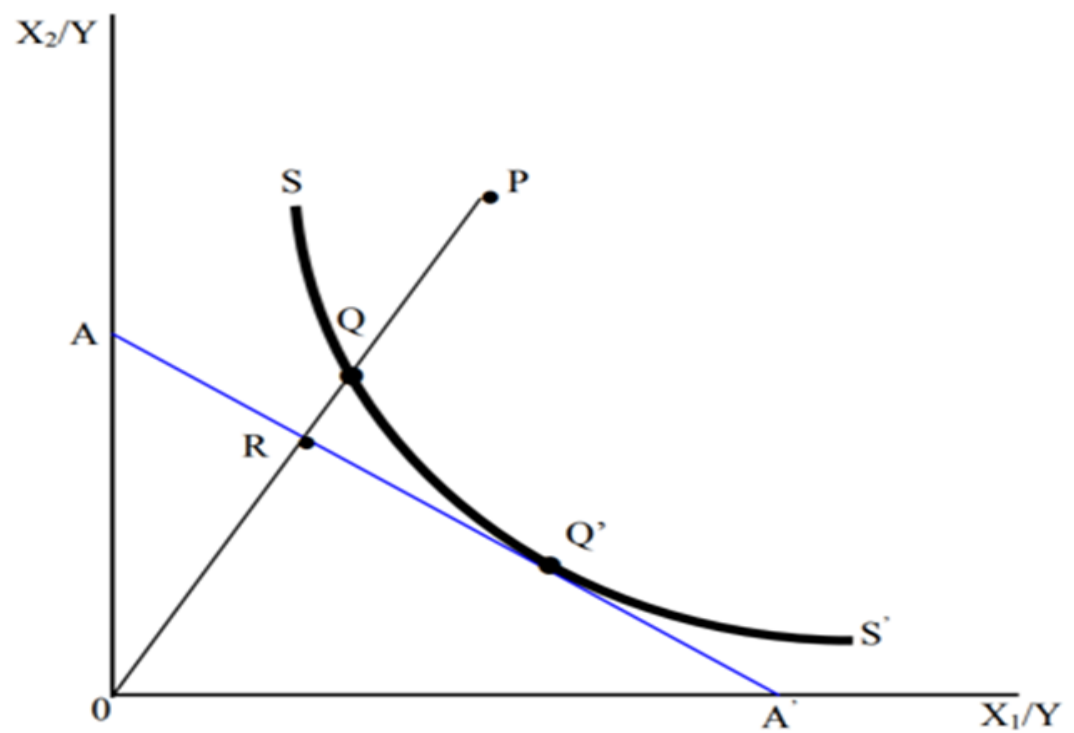

Hình 1. Minh hoạ cách tính TE và $\mathrm{AE}$ 
Có nhiều nghiên cứu về hiệu quả kinh tế trong sản xuất nông nghiệp của các tác giả trong và ngoài nước như cây khóm (N. Q. Nguyen \& Mai, 2015), cây cam (T. T. T. Nguyen et al., 2020), cây ô liu (Fernández-Uclés et al., 2020), cây lúa (T. T. Nguyen, Huynh, Vo, \& Tran, 2018), cây sen (T. V. Nguyen \& Pham, 2014), cây xoài (Emmanuel \& John, 2017), cây dâu và cây cà chua (Jung \& Yang, 2016), cây táo, cây lê và cây nho (Lee, Yun, Kim, \& Yang, 2015), dưa hấu (Doan \& Do, 2016), cây ca cao (Danso-Abbeam, Baiyegunhi, \& Ojo, 2020), cây táo (Ma, Renwick, Yuan, \& Ratna, 2018), cà chua bi (Yang, Zhu, \& Wang, 2020), cây cam sành (D. T. A. Tran, Quan, \& Thach, 2017). Các nghiên cứu đều sử dụng phương pháp màng bao dữ liệu (DEA - Data Envelopment Analysis) để ước lượng hiệu quả kỹ thuật, hiệu quả phân phối theo phương pháp phi tham số và các yếu tố đầu vào để đo lường hiệu quả kinh tế là giống, phân bón, thuốc BVTV, công lao động và đầu ra sản xuất là năng suất cây trồng.

\section{Phương pháp nghiên cứu}

\subsection{Nguồn số liệu}

Theo nghiên cứu của Tabachnick và Fidell (1996) khi sử dụng các phương pháp hồi quy, kích thước mẫu cần thiết được tính theo công thức: $\mathrm{n} \geq 50+8 \mathrm{p}$. Trong đó: $\mathrm{n}$ là kích thước mẫu tối thiểu cần thiết, $p$ là số lượng biến độc lập trong mô hình. Do đó, 05 biến độc lập trong mô hình nghiên cứu được đề xuất thì cỡ mẫu cần điều tra là $\mathrm{n} \geq 50+8 * 5=90$ quan sát. Vậy với cỡ mẫu 240 quan sát, dữ liệu đã đảm bảo thực hiện kiểm định mô hình nghiên cứu. Số liệu sơ cấp được thu thập bằng phương pháp chọn mẫu ngẫu nhiên và tiến hành phỏng vấn trực tiếp các nông hộ trồng táo (gồm có 101 hộ có áp dụng mô hình nhà lưới và 139 hộ không áp dụng mô hình nhà lưới) tại huyện Ninh Phước, tỉnh Ninh Thuận. Đây là một trong bốn địa phương có diện tích trồng táo lớn nhất tỉnh. Ngoài ra, kết hợp với các thông tin thứ cấp từ nhiều nguồn khác nhau, bao gồm các tài liệu, các báo cáo, các nghiên cứu trong và ngoài nước được thu thập để phục vụ cho nghiên cứu. Các thông tin đã thu thập được tổng hợp, tính toán và phân tích bằng phần mềm Excel và DEA 4.1.

\subsection{Phương pháp phân tích và xử lý số liệu}

Nhằm đo lường hiệu quả kinh tế trong sản xuất táo của nông hộ thì phương pháp màng bao dữ liệu hay còn gọi là phương pháp DEA sẽ được sử dụng trong nghiên cứu. Phương pháp DEA được vận dụng bởi vì DEA dựa vào kỹ thuật chương trình tuyến tính toán học để ước lượng cận biên sản xuất chứ không yêu cầu phải xác định một dạng hàm cụ thể và có thể thực hiện trong phạm vi cỡ mẫu nhỏ (Coelli, Rao, O’Donnell, \& Battese, 2005). Mặt khác, các chỉ tiêu hiệu quả chi phí (Cost Efficiency - CE) và hiệu quả kỹ thuật (Technical Efficiency - TE) sẽ được tính toán theo mô hình kinh tế lượng của Rios và Shively (2005). Hiệu quả kỹ thuật và hiệu quả chi phí được thể hiện như sau:

Hiệu quả kĩ thuật (1)

$$
\begin{gathered}
\underset{y, \lambda^{\prime}, \ldots, \lambda^{k}}{\max } y \\
\text { Ràng buộc: } \quad \sum_{k=1}^{K} y^{k} \lambda^{k} \geq y \\
\sum_{k=1}^{K} \lambda^{k}=1 \\
\lambda^{k} \geq 0
\end{gathered}
$$

Hiệu quả chi phí (2)

$$
\min _{x_{1} \rightarrow x_{j, \lambda}} \sum_{n=1}^{t} w_{n}^{0} x_{n}
$$

Ràng buộc: $\sum_{k=1}^{K} y^{k} \lambda^{k} \geq y$

$$
\begin{aligned}
& \sum_{k=1}^{K} x_{n}^{k} \lambda^{k} \leq x_{n} \text { với } 1 \leq n \leq t \\
& \sum_{k=1}^{K} x_{n}^{k} \lambda^{k} \leq x_{n}^{0} \text { với } n>t
\end{aligned}
$$


Trong đó

$y$ là giá trị sản lượng táo tối ưu

$y^{k}$ là giá trị sản lượng táo của hộ thứ $\mathrm{k}$

$x_{n}^{k}$ là chi phí đầu vào thứ $\mathrm{n}$ sử dụng tại hộ k

$x_{n}^{0}$ yếu tố chi phí đầu vào thứ $\mathrm{n}$ sử dụng tại hộ đang kiểm định hiệu quả kỹ thuật

$\lambda^{\mathrm{k}}$ là trọng số gán cho hộ thứ $\mathrm{k}$

$$
\sum_{k=1}^{K} \lambda^{k}=1 \text { và } \lambda^{k} \geq 0
$$

Trong đó

$w_{n}^{0}$ là chi phí của yếu tố đầu vào thứ $\mathrm{n}$ ( $\mathrm{n}$ $=1 \ldots . \mathrm{t})$ của hộ sản xuất táo

$\lambda^{\mathrm{k}}$ là trọng số gán cho hộ thứ $\mathrm{k}$ để thành lập vectơ yếu tố đầu vào

$x_{n}$ là chi phí đầu vào tối ưu $\mathrm{n}(\mathrm{n}=1 \ldots ., \mathrm{t})$

$y^{k}$ là giá trị sản lượng của hộ thứ $\mathrm{k}(\mathrm{k}=1 \ldots \mathrm{K})$

$x_{n}^{k}$ là chi phí đầu vào cho hộ thứ $\mathrm{k}$

$x_{n}^{0}$ là chi phí đầu vào cố định của hộ đang kiểm định về hiệu quả chi phí

Trong đó:

$$
\begin{aligned}
& \mathrm{Y}_{\mathrm{j}} \text { : Năng suất táo }\left(\mathrm{kg} / 1,000 \mathrm{~m}^{2} / \mathrm{vụ}\right) \\
& \mathrm{X}_{1} \text { : Lượng phân vô cơ }\left(\mathrm{kg} / 1,000 \mathrm{~m}^{2} / \mathrm{vụ}\right) \\
& \mathrm{X}_{2} \text { : Lượng phân hữu cơ }\left(\mathrm{kg} / 1,000 \mathrm{~m}^{2} / \mathrm{vu}\right) \\
& \mathrm{X}_{3} \text { : Lượng thuốc bvtv }\left(1 \text { ít } / 1,000 \mathrm{~m}^{2} / \mathrm{vụ}\right) \\
& \mathrm{X}_{4} \text { : Lượng nước tưới }\left(\mathrm{m}^{3} / 1,000 \mathrm{~m}^{2} / \mathrm{vu}\right) \\
& \mathrm{X}_{5} \text { : Công lao động (ngày công lao động/1,000m²/vụ) }
\end{aligned}
$$

Chỉ số hiệu quả kỹ thuật (TE) chính là tỷ số giữa năng suất sản xuất táo thực tế của nông hộ $\left(\mathrm{y}^{0}\right)$ trên năng suất sản xuất táo tối đa $(\mathrm{y})$. Những hộ sản xuất táo được xem là hiệu quả về mặt kỹ thuật là những hộ có chỉ số $\mathrm{TE}$ bằng 1 , và những hộ gia đình sản xuất táo không có hiệu quả về mặt kỹ thuật là những hộ có chỉ số TE nhỏ hơn 1. Chỉ số hiệu quả về chi phí được tính bằng tỷ số giữa mức chi phí tối ưu $\left(w_{n}^{0} x_{n}\right)$ và chi phí quan sát của hộ sản xuất táo thứ $\mathrm{k}\left(w_{n}^{0} x_{n}^{k}\right)$.

\section{Kết quả và thảo luận}

\subsection{Hiệu quả tài chính trong sản xuất táo của nông hộ tại huyện Ninh Phước}

\subsubsection{Một số đặc điểm về nhân khẩu học và xã hội học của hộ điều tra}

Bảng 1 thể hiện các giá trị thống kê mô tả, sự khác biệt trung bình giữa hai nhóm hộ áp dụng và không áp dụng mô hình bao lưới. Phần lớn chủ hộ là nam giới với hộ áp dụng $(75.2 \%)$ và nhóm không áp dụng $(60.4 \%)$. Điều này là hợp lý vì sản xuất nông nghiệp đòi hỏi sức mạnh thể chất nhiều hơn. Thêm vào đó, nhóm hộ áp dụng mô hình có độ tuổi trong khoảng 50 - 60 tuổi (84.2\%). Nhưng nhóm hộ không áp dụng mô hình từ 40 - 50 tuổi (61.1\%). Cả hai nhóm hộ ở độ tuổi này vẫn còn đảm bảo sức khỏe để tham gia sản xuất.

Mặt khác, trình độ học vấn của cả hai nhóm hộ chủ yếu là trung học cơ sở với nhóm hộ áp dụng (54.5\%) và nhóm hộ không áp dụng (48.9\%), đồng thời, kinh nghiệm của nông hộ từ 5-10 năm của nhóm áp dụng (44.6\%), nhóm không áp dụng (43.9\%). Điều này là một thuận lợi cho các nông hộ trong việc tiếp thu kiến thức và kĩ thuật trong sản xuất. Diện tích sản xuất táo trung bình ở hai nhóm hộ có qui mô từ $1,000 \mathrm{~m}^{2}-5,000 \mathrm{~m}^{2}$ với nhóm hộ áp dụng $(75.3 \%)$ và hộ không áp dụng $(71.9 \%)$. 


\section{Bảng 1}

Thông tin chung về đối tượng phỏng vấn

\begin{tabular}{|c|c|c|c|c|}
\hline \multirow{2}{*}{ Chỉ tiêu } & \multicolumn{2}{|c|}{$\begin{array}{l}\text { Nhóm hộ áp dụng } \\
\text { mô hình nhà lưới }\end{array}$} & \multicolumn{2}{|c|}{$\begin{array}{c}\text { Nhóm hộ không áp dụng } \\
\text { mô hình nhà lưới }\end{array}$} \\
\hline & $\begin{array}{c}\text { Tần số } \\
\text { (Hộ) }\end{array}$ & $\begin{array}{l}\text { Tỷ lệ } \\
(\%)\end{array}$ & $\begin{array}{c}\text { Tần số } \\
\text { (Hộ) }\end{array}$ & $\begin{array}{l}\text { Tỷ lệ } \\
(\%)\end{array}$ \\
\hline \multicolumn{5}{|l|}{ 1. Giới tính chủ hộ } \\
\hline Nam & 76 & 75.2 & 84 & 60.4 \\
\hline Nũ̃ & 25 & 24.8 & 55 & 39.6 \\
\hline \multicolumn{5}{|l|}{ 2. Tuổi chủ hộ } \\
\hline$<=30$ tuổi & 3 & 3.0 & 7 & 5.0 \\
\hline 30 tuổi - 40 tuổi & 20 & 19.8 & 25 & 18.0 \\
\hline 40 tuổi - 50 tuổi & 29 & 28.7 & 53 & 38.1 \\
\hline 50 tuổi - 60 tuổi & 33 & 32.7 & 37 & 26.6 \\
\hline$>60$ tuổi & 16 & 15.8 & 17 & 12.2 \\
\hline \multicolumn{5}{|l|}{ 3. Trình độ học vấn } \\
\hline Mù chữ & 1 & 1.0 & 3 & 2.2 \\
\hline Tiểu học & 20 & 19.8 & 39 & 28.1 \\
\hline Trung học cơ sở & 55 & 54.5 & 68 & 48.9 \\
\hline Trung học phổ thông & 22 & 21.8 & 27 & 19.4 \\
\hline Cao đẳng - Đại học & 3 & 2.9 & 2 & 1.4 \\
\hline \multicolumn{5}{|l|}{ 4. Kinh nghiệm } \\
\hline$<=05$ năm & 22 & 21.8 & 39 & 28.1 \\
\hline 05 năm - 10 năm & 45 & 44.6 & 61 & 43.9 \\
\hline 10 năm - 15 năm & 25 & 24.8 & 27 & 19.4 \\
\hline 15 năm - 20 năm & 8 & 7.9 & 10 & 7.2 \\
\hline$>20$ năm & 1 & 0.9 & 2 & 1.4 \\
\hline \multicolumn{5}{|l|}{ 5. Qui mô sản xuất } \\
\hline$<=1,000 \mathrm{~m}^{2}$ & 10 & 9.9 & 27 & 19.4 \\
\hline $1,000 m^{2}-5,000 m^{2}$ & 76 & 75.3 & 100 & 71.9 \\
\hline $5,000 m^{2}-10,000 m^{2}$ & 8 & 7.9 & 11 & 7.9 \\
\hline$>10,000 \mathrm{~m}^{2}$ & 7 & 6.9 & 1 & 0.8 \\
\hline
\end{tabular}

Nguồn: Số liệu điều tra (2020) 


\subsubsection{So sánh hiệu quả tài chính trong sản xuất táo giũa hai nhóm hộ}

Từ kết quả tại Bảng 2 cho thấy, chi phí sản xuất táo của nông hộ trung bình là 18,704 triệu đồng $/ 1,000 \mathrm{~m}^{2} / v u ̣$ trong đó nhóm hộ áp dụng mô hình có chí phí sản xuất nhỏ hơn các hộ không áp dụng mô hình. Theo đó, hiệu quả tài chính của nông hộ trong sản xuất táo có áp dụng mô hình (lợi nhuận/chi phí là 1.87 lần và thu nhập/chi phí là 2.13 lần) cao hơn so với nông hộ không áp dụng mô hình (lợi nhuận/chi phí là 0.66 lần và thu nhập/chi phí là 0.93 lần). Điều này phần nào lý giải tầm quan trọng của mô hình nhà lưới trong sản xuất táo hiện nay.

\section{Bảng 2}

So sánh hiệu quả trong sản xuất táo giữa hai nhóm hộ

\begin{tabular}{|c|c|c|c|}
\hline Chỉ tiêu & ĐVT & $\begin{array}{l}\text { Nhóm hộ áp dụng } \\
\text { mô hình nhà lưới }\end{array}$ & $\begin{array}{l}\text { Nhóm hộ không áp } \\
\text { dụng mô hình nhà lưới }\end{array}$ \\
\hline 1. Chi phí sản xuất & $1,000 \mathrm{~d} / 1,000 \mathrm{~m}^{2}$ & 17,841 & 19,283 \\
\hline Chi phí vật chất & $1,000 \mathrm{~d} / 1,000 \mathrm{~m}^{2}$ & 11,797 & 12,280 \\
\hline Chi phí phân bón & $1,000 \mathrm{~d} / 1,000 \mathrm{~m}^{2}$ & 6,117 & 7,072 \\
\hline Chi phí thuốc BVTV & $1,000 \mathrm{~d} / 1,000 \mathrm{~m}^{2}$ & 740 & 2,364 \\
\hline Chi phí nước & $1,000 \mathrm{~d} / 1,000 \mathrm{~m}^{2}$ & 1,680 & 1,754 \\
\hline Chi phí khấu hao & $1,000 \mathrm{~d} / 1,000 \mathrm{~m}^{2}$ & 2,540 & 465 \\
\hline Chi phí khác & $1,000 \mathrm{~d} / 1,000 \mathrm{~m}^{2}$ & 720 & 625 \\
\hline Chi phí lao động & & 6,044 & 7,003 \\
\hline Chi phí lao động nhà & $1,000 \mathrm{~d} / 1,000 \mathrm{~m}^{2}$ & 4,644 & 5,203 \\
\hline Chi phí lao động thuê & $1,000 \mathrm{~d} / 1,000 \mathrm{~m}^{2}$ & 1,400 & 1,800 \\
\hline \multicolumn{4}{|l|}{ 2. Kết quả sản xuất } \\
\hline Doanh thu & $1,000 \mathrm{~d} / 1,000 \mathrm{~m}^{2}$ & 51,218 & 31,945 \\
\hline Lợi nhuận & $1,000 \mathrm{~d} / 1,000 \mathrm{~m}^{2}$ & 33,377 & 12,662 \\
\hline Thu nhập & $1,000 \mathrm{~d} / 1,000 \mathrm{~m}^{2}$ & 38,021 & 17,865 \\
\hline \multicolumn{4}{|l|}{ 3. Hiệu quả kinh tế } \\
\hline Lợi nhuận/chi phí & Lần & 1.87 & 0.66 \\
\hline Thu nhập/chi phí & Lần & 2.13 & 0.93 \\
\hline Doanh thu/chi phí & Lần & 2.87 & 1.65 \\
\hline
\end{tabular}

Nguồn: Số liệu điều tra (2020)

\subsection{Phân tích hiệu quả kinh tế trong sản xuất táo của nông hộ tại huyện Ninh Phước}

Kết quả tại Bảng 3 mô tả hiệu quả kỹ thuật, hiệu quả phân phối và hiệu quả chi phí (hiệu quả kinh tế) của hai nhóm hộ. Các chỉ số của nhóm hộ áp dụng mô hình nhà lưới đều cao hơn so với nhóm hộ không áp dụng mô hình nhà lưới, với hiệu quả kỹ thuật đạt từ 0.7 trở lên là 101 hộ (áp dụng mô hình) trong đó 67 hộ có hiệu quả kỹ thuật trên 0.9, hiệu quả kỹ thuật trung bình của nhóm hộ áp dụng mô hình là rất cao 0.96 so với mức hiệu quả kỹ thuật trung bình của nhóm hộ không áp dụng mô hình là 0.67 (trong đó 39 hộ đạt hiệu quả kỹ thuật từ 0.7 trở lên nhưng không 
có hộ nào đạt hiệu quả kỹ thuật trên 0.9). Tuy nhiên, việc kết hợp các yếu tố đầu vào chưa hợp lý nên hiệu quả phân phối không cao ở hai nhóm hộ, hiệu quả phân phối trung bình là 0.71 (nhóm hộ áp dụng mô hình) và 0.51 (nhóm hộ không áp dụng mô hình), số hộ đạt hiệu quả phân phối nguồn lực từ 0.8 trở lên rất ít. Mặt khác, kết quả tính toán còn cho thấy không có hộ nào đạt hiệu quả sử dụng chi phí trong khoảng từ 0.9 đến 1 nhưng nhóm hộ áp dụng mô hình nhà lưới $(0.71)$ vẫn tốt hơn so với nhóm hộ không áp dụng mô hình nhà lưới (0.34).

\section{Bảng 3}

So sánh hiệu quả sản xuất táo giữa hộ áp dụng và không áp dụng

\begin{tabular}{ccccccc}
\hline \multirow{2}{*}{$\begin{array}{c}\text { Giá trị hiệu } \\
\text { quả }\end{array}$} & \multicolumn{2}{c}{ Hiệu quả kỹ thuật } & \multicolumn{2}{c}{ Hiệu quả phân phối } & \multicolumn{2}{c}{ Hiệu quả chi phí } \\
\cline { 2 - 7 } & $\begin{array}{c}\text { Hộ áp } \\
\text { dụng }\end{array}$ & $\begin{array}{c}\text { Hộ không } \\
\text { áp dụng }\end{array}$ & Hộ áp dụng & $\begin{array}{c}\text { Hộ không } \\
\text { áp dụng }\end{array}$ & Hộ áp dụng & $\begin{array}{c}\text { Hộ không } \\
\text { áp dụng }\end{array}$ \\
\hline$<=0.5$ & 0 & 19 & 0 & 7 & 0 & 12 \\
$0.5-0.6$ & 0 & 42 & 0 & 63 & 0 & 37 \\
$0.6-0.7$ & 0 & 39 & 9 & 52 & 41 & 65 \\
$0.7-0.8$ & 14 & 25 & 72 & 13 & 52 & 23 \\
$0.8-0.9$ & 20 & 14 & 20 & 4 & 8 & 2 \\
$0.9-1$ & 67 & 0 & 0 & 0 & 0 & 0 \\
\hline Trung bình & $\mathbf{0 . 9 6 2}$ & $\mathbf{0 . 6 7 4}$ & $\mathbf{0 . 7 4 1}$ & $\mathbf{0 . 5 1 6}$ & $\mathbf{0 . 7 1 3}$ & $\mathbf{0 . 3 4 8}$ \\
\hline
\end{tabular}

Nguồn: Kết quả phân tích DEA (2020)

Kết quả phân tích DEA về lượng yếu tố đầu vào bị lãng phí trong sản xuất táo giữa hai nhóm hộ được thể hiện tại Bảng 4 , dựa vào kết quả này thì hai nhóm hộ có thể giảm các yếu tố đầu vào để đạt được mức năng suất hiện tại. Nhóm hộ không áp dụng mô hình nhà lưới lãng phí hơn nhóm hộ áp dụng mô hình nhà lưới, đặc biệt là lãng phí trong sử dụng thuốc bảo vệ thực vật gấp 05 lần $(160$ - 360 lít/1,000m² $)$ và công lao động gấp 03 lần $\left(02-06\right.$ ngày/1,000 $\left.\mathrm{m}^{2}\right)$. Điều này là do trong mô hình nhà lưới sẽ hạn chế tối đa sự tấn công của sâu bệnh, chim, côn trùng nên lượng yếu tố đầu vào được kiểm soát tốt hơn nên lượng thuốc BVTV được sử dụng khá ít.

\section{Bảng 4}

Lượng lãng phí các yếu tố đầu vào của hộ áp dụng và hộ không áp dụng

\begin{tabular}{lcccc}
\hline \multirow{2}{*}{ Đầu vào } & \multicolumn{2}{c}{ Thực tế } & \multicolumn{2}{c}{ Lãng phí } \\
& Hộ & Hộ không & Hộ & Hộ không \\
& áp dụng & áp dụng & áp dụng & ạng \\
\hline Phân vô cơ $\left(\mathrm{kg} / 1,000 \mathrm{~m}^{2}\right)$ & 231 & 291 & 16 & 43 \\
Phân hữu cơ $\left(\mathrm{kg} / 1,000 \mathrm{~m}^{2}\right)$ & 1,220 & 1,641 & 160 & 360 \\
Thuốc bvtv $\left(\mathrm{lí}^{2} / 1,000 \mathrm{~m}^{2}\right)$ & 1.20 & 2.30 & 0.15 & 0.8 \\
Lượng nước tưới $\left(\mathrm{m}^{3} / 1,000 \mathrm{~m}^{2}\right)$ & 33.6 & 35.1 & 4 & 7 \\
Công lao động $\left(\mathrm{ngày} / 1,000 \mathrm{~m}^{2}\right)$ & 36 & 43 & 2 & 6 \\
\hline
\end{tabular}

Nguồn: Kết quả phân tích DEA (2020) 
Kết quả phân tích tại Bảng 5 cho thấy sự khác biệt về hiệu quả theo quy mô giữa hộ áp dụng và hộ không áp dụng mô hình nhà lưới. Do mô hình nhà lưới trong canh tác táo của nông hộ mới được áp dụng trong thời gian gần đây nên số hộ đang trong khu vực tăng theo quy mô sản xuất (61.39\%) cao hơn nhiều so với hộ không áp dụng (25.9\%). Điều này cho thấy, nếu hộ áp dụng đầu tư sản xuất táo theo mô hình nhà lưới có thể đạt được năng suất táo tối đa và tỷ lệ hộ phải giảm quy mô sản xuất cũng ít hơn. Mặt khác, đa số hộ canh tác táo không áp dụng mô hình đang trong khu vực giảm theo quy mô (quy mô nhỏ hơn mức tối ưu) chiếm đến $46.04 \%$ và nhóm hộ đạt tối ưu về quy mô (không đổi theo quy mô) giữa hai nhóm hộ là tương đương nhau với $25.74 \%$ (hộ áp dụng), 28.06\% (hộ không áp dụng).

\section{Bảng 5}

Hiệu quả quy mô của hộ áp dụng và hộ không áp dụng

\begin{tabular}{lcccc}
\hline \multirow{2}{*}{ Hiệu quả theo quy mô sản xuất - } & \multicolumn{2}{c}{ Hộ áp dụng } & \multicolumn{2}{c}{ Hộ không áp dụng } \\
\cline { 2 - 5 } & Số hộ & Tỷ lệ (\%) & Số hộ & Tỷ lệ (\%) \\
\hline Hiệu quả tăng theo quy mô (IRS) & 62 & 61.39 & 36 & 25.90 \\
Hiệu quả giảm theo quy mô (DRS) & 13 & 12.87 & 64 & 46.04 \\
Hiệu quả không đổi theo quy mô (CRS) & 26 & 25.74 & 39 & 28.06 \\
\hline
\end{tabular}

Nguồn: Kết quả phân tích DEA (2020)

\section{3. Đề xuất một số khuyến nghị nhằm nâng cao hiệu quả trong sản xuất táo của nông hộ}

Từ kết quả nghiên cứu thì để nâng cao hiệu quả kinh tế trong sản xuất táo của nông hộ thì một số khuyến nghị được đề xuất như sau:

Tổ chức các lớp tập huấn khuyến nông nhằm đánh giá hiệu quả mô hình nhà lưới mang lại, giúp nông hộ hiểu rõ về lợi ích mô hình, tiếp thu và áp dụng công nghệ mới vào sản xuất sẽ dễ dàng, cũng là cơ sở để nhân rộng mô hình nhà lưới.

Xây dựng chính sách hỗ trợ tài chính từ địa phương. Địa phương nên hỗ trợ một phần hoặc toàn bộ kinh phí cho nông hộ làm nhà lưới, vì nông hộ còn hạn chế trong vấn đề tài chính nên đầu tư nhà lưới không đủ chuẩn. Mặt khác, khuyến khích các doanh nghiệp cùng tham gia vào mô hình này để giúp cho nông hộ không phải lo làm nhà lưới riêng lẻ mà do chính các doanh nghiệp đầu tư trước cho nông dân.

\section{Kết luận}

Mô hình nhà lưới trong canh tác táo là phương pháp bảo vệ hiệu quả trước sự tấn công của sâu bệnh. Bài báo đã phân tích hiệu quả kinh tế trong sản xuất táo theo mô hình nhà lưới của nông hộ tại huyện Ninh Phước, tỉnh Ninh Thuận. Kết quả nghiên cứu cho thấy, khi áp dụng mô hình nhà lưới thì nông hộ trồng táo tăng hiệu quả tài chính gấp 1.87 lần so với hộ không áp dụng và giảm chi phí sản xuất cũng như giảm lượng lãng phí các yếu tố đầu vào trong quá trình sản xuất. Mặt khác, phương pháp phân tích màng bao dữ liệu (DEA) cho thấy hiệu quả kinh tế trong canh tác táo theo mô hình nhà lưới là tương đối cao với hiệu quả kỹ thuật là 0.962 , hiệu quả phân phối nguồn lực là 0.741 và hiệu quả sử dụng chi phí là 0.713 so với hộ không áp dụng chỉ đạt hiệu quả kỹ thuật là 0.674 , hiệu quả phân phối nguồn lực là 0.516 và hiệu quả sử dụng chi phí là 0.348 . Mặc dù hiệu quả kinh tế vẫn chưa đạt được kết quả tối ưu trong cả hai nhóm hộ nhưng kết quả đã chỉ ra sự khác biệt về hiệu quả kinh tế giữa nhóm hộ áp dụng và không áp dụng mô hình nhà lưới trong sản xuất táo tại huyện Ninh Phước, tỉnh Ninh Thuận. 


\section{Tài liệu tham khảo}

Ali, M., \& Byerlee, D. (1991). Technical efficiency of rice farmars in irrigated, rainfed low-land anh upland environments: A frontier production function analysis. Philippine Journal of Crop Science, 18(2), 59-69.

Coelli, T. J., Rao, D. S. P., O’Donnell, C. J., \& Battese G. E. (2005). An introduction to efficiency and productivity analysis (2nd ed.). Dordrecht, Netherlands: Kluwer Academic Publishers.

Danso-Abbeam, G., Baiyegunhi, L. J., \& Ojo, T. O. (2020). Gender differentials in technical efficiency of Ghanaian cocoa farms. Heliyon, 6(5), Article e04012.

Doan, N. H., \& Do, X. V. (2016). Phân tích hiệu quả kỹ thuật, hiệu quả phân phối nguồn lực của hộ trồng dưa hấu tại huyện Phong Điền, thành phố Cần Thơ năm 2014 [Technical, allocative and cost efficiencies of watermelon farming households in Phong Dien district, Can Tho City in 2014]. Tạp chí Khoa học Trưòng Đại học Cần Tho, 42(D), 9-14.

Emmanuel, W. I., \& John, A. M. (2017). Estimating economic efficiency of mango production in Ghana. ADRRI Journal of Agriculture and Food Sciences, 3(7), 29-46.

Farrell, M. J. (1957). The measurement of productive efficiency. Journal of the Royal Statistical Society: Series A (General), 120(3), 253-281.

Fernández-Uclés, D., Elfkih, S., Mozas-Moral, A., Bernal-Jurado, E., Medina-Viruel, M. J., \& Abdallah, S. B. (2020). Economic efficiency in the Tunisian olive oil sector. Agriculture, 10(9), Article 391.

Jung, D. E., \& Yang, S. R. (2016). An analysis of economic efficiency of fruits and vegetables farms: The case of strawberries and tomatoes farms. Korean Journal of Organic Agriculture, 24(3), 385-412.

Lee, C., Yun, S. J., Kim, G., \& Yang, S. R. (2015). An analysis of economic efficiency of fruits farms: The case of apples, pears, and grapes farms. Korean Journal of Organic Agriculture, 23(4), 615-641.

Ma, W., Renwick, A., Yuan, P., \& Ratna, N. (2018). Agricultural cooperative membership and technical efficiency of apple farmers in China: An analysis accounting for selectivity bias. Food Policy, 81, 122-132. doi:10.1016/j.foodpol.2018.10.009

Nguyen, N. Q., \& Mai, N. V. (2015). Đánh giá hiệu quả sản xuất khóm của nông hộ ở huyện Tân Phước, tỉnh Tiền Giang [Evaluate the productive efficiency of pineapples growing households in Tan Phuoc District, Tien Giang Province]. Tạp chí Khoa hoc Truòng Đại hoc Cần Tho, 36(D), 1-9.

Nguyen, T. T. T., Le, H. H., Ho, H. T. M., Dogot, T., Burny, P., Bui, N. T., \& Lebailly, P. (2020). Efficiency analysis of the progress of orange farms in Tuyen Quang province, Vietnam towards sustainable development. Sustainability, 12(8), Article 3170. doi: $10.3390 /$ su 12083170

Nguyen, T. T., Huynh, K. V., Vo, T. H., \& Tran, H. M. (2018). Phân tích hiệu quả kinh tế mô hình lúa - tôm tại huyện An Biên, tỉnh Kiên Giang [Economic efficiency analysis of rice-shrimp farming in An Bien district, Kien Giang province]. Tạp chí Khoa họ Trường Đại họ Cần Tho; 54(9D), 149-156. doi:10.22144/ctu.jvn.2018.191

Nguyen, T. V., \& Pham, T. L. (2014). Phân tích hiệu quả kinh tế của nông hộ trồng sen trên địa bàn tỉnh Đồng Tháp [Analyzing economic efficiency of the lotus farms in Dong Thap 
Province]. Tạp chí Khoa họ Truờng Đại hoc Cần Tho, 30(D), 120-128.

Rios, A. R., \& Shively, G. E. (2005). Farm size and nonparametric efficiency measurements for coffee farms in Vietnam. Paper presented at the American Agricultural Economics Association Annual meeting, Providence, Rhode Island.

Sở NN \& PTNT tỉnh Ninh Thuận. (2020). Báo cáo tình hình và kết quả thực hiện 9 tháng đầu năm 2019, nhiệm vu trọng tâm và giải pháp thục hiện 3 tháng cuối năm 2019 của Ngành nông nghiẹp [Report on the situation and performance results of the first 9 months of 2019, key tasks and solutions for the last 3 months of 2019 of the Agriculture sector]. Retrieved February 10, 2021, from https://www.ninhthuan.gov.vn/chinhquyen/sonnnt/Admin/Lich\% 202019/BC\%20493(19.09.2019_14h35p04)_signed.pdf

Tabachnick, B. G., \& Fidell, L. S. (1996). Using multivariate statistics (3rd ed.). New York, NY: HarperCollins.

Tran, D. T. A., Quan, N. M., \& Thach, K. K. (2017). Phân tích hiệu quả kỹ thuật của nông hộ sản xuất cam sảnh ở huyện Cái Bè, tỉnh Tiền Giang. [Technical efficiency of king mandarin production in Cai Be dictrict, Tien Giang province]. Tạp chí Khoa hoc Trường Đại hoc Cần Tho, 48(D), 112-119. doi:10.22144/ctu.jvn.2017.636

Tran, N. H., \& Le, T. T. H. (2019). Tác động của hạn hán đến khả năng sản xuất tỏi của nông hộ tại huyện Ninh Hải, tỉnh Ninh Thuận [The impact of drought in adaptability of farmer's garlic cultivation in Ninh Hai district, Ninh Thuan province]. Tạp chí Khoa họ và Công nghẹ Lâm Nghiệp, 6, 170-176.

Yang, Q., Zhu, Y., \& Wang, J. (2020). Adoption of drip fertigation system and technical efficiency of cherry tomato farmers in Southern China. Journal of Cleaner Production, 275, Article 123980. doi:10.1016/j/clepro.2020.123980 
